\title{
Métodos y Aplicaciones para el Mapeo de Internet
}

\author{
Fran cisco Manuel Mateos Rodríguez \\ FacultaddeBiblioteconomíayDocumentación, \\ Universidad de Granada \\ E-Mail:frank50@latinmail.com
}

\begin{abstract}
RESUMEN
Sepresen ta una re vi sión de al gu nas delas ini cia tivas y sis te mas que se pro ponen des cribir las téc ni cas em plea das para vi sua li zar la in for ma ción. Se mues tra una tipolo gía de he rra mien tas de análi sis y sus apli ca cio nes, en su ma yo ría simplesdesarrollosexperimentalesyprototípicos que sin embargo son considerados como herramien tas depodero sautilidad paraope rarda tos relacionales y com pren der los pa tro nes es truc tu ra les delas rela cio nes en treins titur ciones y el análisis de redes sociales.

Palabras clave:Visualización de información; Redes sociales; Representación de redes; Análisis de redes; Representación de informaciones; información gráfica; Técnicas de visualización
\end{abstract}

\section{METHODS AND APPLI CATIONSFOR INTER NET MAPPING Fran CISCOMANUEL MATEOS-RODRÍGUEZ}

\begin{abstract}
The pres ent ar ti cle re views some of the proj ects and sys tems pro posed for infor ma tion visu aliza tion and de scribes the tech niques used. A ty pology of the tools used for analy sis and their ap pli ca tions is also pre sented. Many of these are simple ex perimen tal de velop ments and pro to types, none theless they are consid ered pow er ful tools in rela tional data op era tions for the un derstand ing of structural patterns between institutions and in social network analysis.

Keywords: Information Visualization; Social Networks; Network Representation; Network Análysis; Information Representation; Graphic Information; Visualization Techniques.
\end{abstract}

\section{INTRODUCCIÓN}

L a visua lización delain for ma ción es una ac tividad que el hom bre havenido cul tivando con bastante antelación a la aparición de los ordenadores, no obstante, sines tos últimos mu chas aplica ciones devisualiza ción seríanim posibles. La visualización toma comúnmente la forma de gráficos, figuras e imágenes que son utilizados para apreciar fenómenos naturales (p.ej. mapas meteorológicos), estructuras teóricas e invisibles (p. ej. partículas atómicas y subatómicas), flujos de diferentes 


\section{InvestigacoónBiblictecoógia v. 17 N o. 35 julio/ diciembre de 2003}

for mas de ma te ria (p. ej. flu jo de aire por el ala de un avión), sis te mas de in te rac ciones (p.ej.proce sos químicos), mo delos de rela ciónen se ries mul tiva rian tes de da tos (p. ej. datos médicos y sociométricos), entre otros fenómenos. Recientemente, se están aplicando estas técnicas a series de datos en el mundo de los negocios y a los contenidos informativos de conjuntos de documentos (Williams, Sochats y Morse 1995). ${ }^{1}$ Esta última aplicación es la que a nosotros nos interesa.

Yes que, des de que se acu ña se el tér mino cibe res pa cio, mu chos han sido los que han sen tidola ne cesidad y han in tentadodarle forma ala red de redes. A represen tar grá fi ca men te In ternet, al he cho de car to gra fiar lo vir tual, sele de no mina hoy día cibergeografía.

La cibergeografía es un campoincipiente que atrae la atención de investigadores provenientes de disciplinas tan diversas como la ingeniería y las ciencias sociales. Estanuevacien cia tieneporcometidoeles tu diodelciberespacio, elámbitoque abarcanlas re des in for máticas, y so bre todo In ternet. Pero ade más, cual quier es pa cio in formático que haya detrás de la pantalla de nuestro ordenador. De hecho, engloba unagranvarie dadde fenómenosgeográ ficoscomolain fraes tructura delatecnología delain formación, las tele comunicaciones, elespacioporelque dis currenlas corrientes de da tos que se trans mi ten con ti nua men te yla de mo gra fía de las nue vas co mu nidadesciberespaciales.

A finde cuen tas, la cibergeo gra fía va a tra tarde percibir, de li mi tary visualizarestos nue vos es pa cios digitales, igual que hace la geo gra fía con el es pa cio físico. Aligualque losmapasgeográ ficos, los ciberma pasayudanalusuario anavegarporlos nuevos territo rios elec tró ni cos, y les fa cili tan la visua liza ción y com pren sión de las nuevas superficies digitales en las redes globales de comunica ciones yen los am plios re cursos deinformaciónenlínea.

Laprincipaldificultad conestetipodevisualización, consiste enimplementaruna representación que muestre las caracterís ticasmásrelevantes de los da tos de un determinadodominio. Estetrabajoconsisteen trans formarda tosabstrac tosyfenómenos com ple jos de la rea li dad en men sajes visibles, lo que hace po si blequelos in divi duos vean "con sus propios ojos" tales datos y fenómenos que son directamente inapren siblesy,portanto, compren danlain forma ción que yaceoculta. Se tratade un actode trans fe ren cia del conocimien to, y porlotan tola visualizaciónse jus tifica por elhechodequeelmundoesmul tifacético, multidimensional,multifenoménico,yde que se pre sen ta como un continum(Tuf te 1990). ${ }^{2}$ Si lo que lla ma mos "la rea li dad" es lo que percibimos, se com pren de que "visuali zar" es un me dio de am pliar el mun do delopercep tible; es de cir, de gene rary trans mi tir co no cimien to que en prin cipiono estáanuestroalcance.

1 Williams, J. G., So chats, K. M., and Mor se, E.V isualization, volu me 30, pages 161-207.In forma tion To day, Inc., Medford, NJ, 1995.

2 Edward Tufte. EnvisianingInfomation Graphics Press, Cheshire Con nec ticut, 1990. 
Ennuestrocaso, ladocumentacióncomienzaamos trarciertointeréspores tos recursos. Whi tey McCain, de la Universidadde Drexel, son quienes más hananaliza do esta ten dencia con cier ta pro fun didad. Poruna parte, para ellos elobje tivo dela ciencia de lain forma ción es el es tu dio delasin ter fa ces en trelagen tey los do cu men toso la lite ra tu ra, y porelotrola ten dencia do minan te con sis te en combinar las visualizaciones (gráficas y por ordenador) con la recuperación automatizada de la información (Whi te y McCain 1997). ${ }^{3}$ Elob je tivo dela vi sua li za ción de tex tos es la trans formacióndel "espacio tex tual" enunanuevarepresentaciónvisualquerevelapatrones temáticos y relaciones entre los documentos de manera natural e intuitiva para el usuario(WhiteyMcCaine1997).

Por ello la re presen ta ción de la in for ma ción de for ma grá fica se con vierteen una herramienta poderosa que facilita su entendimiento y diseminación (Tufte 1990), y constituye también un medio sen cillopararealizarvisualizacio nesdediferentesfenómenos. Esta representación trata de mostrar la realidad que está oculta a simple vis ta, y estololograa tra vés de unametáfo ra, que ya sea de forma ex plícitao im plícita, comunicaundeterminadomensaje.

Elob jetivo delpresen te tra bajo con sis te en revisarbrevementealgunas delasini ciativas y sistemas que utilizan distintas técnicas de visualización de la información con tenida en la red de re des. No obs tan te, sepon drá elén fa sis en aquellasin terfaces que representen en el mo men todelpresente es tu diouna muestra, la cualnopre tende serniremo tamen te ex haus tiva, de aquellas aplica ciones quegenerende forma desa rrollada y comprensible representaciones de informaciónestructurada. Por último, cabe destacar que los sistemas tratados son, en su mayor parte, desarrollos experimentalesyprototípicosqueaúnnohancristalizadoenaplicacionescomerciales.

\section{Visual Site MAP}

Ésta es una aplicación basadaen técnicas deredesneu ro nales artificiales (RNA), desarrolla doporXia Lin, delaUniversidadde Drexel, parala re presenta cióny navega ción de si tios web. Como ya se ha se ña la do la apli ca ción se de no mi na Vi sual Site Mapy trabajacon AppleJavaa tra vésdeunnavega dorweb que soporteestelenguaje. El sistema ana liza uno a uno to dos los en la ces del sitio, re colec tain for ma ción es tadís tica, comola fre cuen cia de pa la bras, y con vier tecada pá gina en unvec tornu mérico. Los vectores serán pos terior men te pro ce sa dos por un tipo es pecial deRNA denominado mapa auto-organizativo o SOM (SAf-OrganizingMap), cuya salida será un mapa bi dimen sio nal al cual se le aña di ráneti que tas en fun ción del análi sis de co-ocur rrenciadelaspalabras.

3 H.D. White,K.W.Mccain. "Visualiza tion oflitera tures", en M.E. Williams(Ed.),AnnualReviend InformationSaienceandTechndogy. Vol. 32, pp. 99-168, 1997. Medford, N J: 


\section{InvestigacónBiblicteedógia v. 17 N o. 35 julio/ diciembre de 2003}

ElVisual Site Map pre sen ta un parde ba rras de po si cio na mien to: uno para pa labras y otro para pun tos. A tra vés del uso de es tas ba rras po dre mosir pro fun di zan do más en los datos visualizados. La barra de palabras nos brinda la posibilidad de ver con mayor o menor detalle las distintas áreas temáticas de las que se compone el mapa en cues tión. Con formeavanza mos la ba rra ha ciala de re cha, iránapa re cien do nuevostérminosquecomplementaránlain forma cióndelcampovisual.Yviceversa, conformenues trodes plazamientolorealicemoshacialaizquierda. Alutilizarlabarra depuntos, podremos dis tinguiren trepun tos rojosque harán refe renciaa en laces externosypuntos azulesquese referirán a enlacesin ternosdel propio si tio web.La aplica ción de este mo delo pare ce seruna delas más prome te do ras en la clasi fica ción aut tomáticamedianteredesneuronales.

De for ma gene ral los dis tin tos ma pas de los si tios web son crea dos tan to por los ad minis tra do res del pro pio si tio como porlos proveedo res de con tenidos, conla finalidadde facilitarlesalos usua riosla ex plora ciónynavega ción de los más complejos espacios web. De igual forma, los mapas web generados mediante esta aplicación también pue den sercrea dos para serutiliza doscomoín dicesdel propiositiowebcon la finalidadde facilitarlesalos propios ad minis tradoresla visualizaciónyelm anejode grandesycomplejaszonasweb.

Deestamanerase pue de establecerqueesta aplicación sebasa en un sistema deorganización que co nec ta y per mi te vi sua lizarla in for ma ción den tro de una red de conocimiento.Esto Permite enlazarficheros, documentos ypáginas web mediantelas dis tin tas subre des y sus aplica cio nes. Se Ilus tra de esta for ma cómo toda la in for ma ción está rela cionada, y se propor cio na un con tex to visualpara do cumen tosy da tos, navegabledeformamuyintuitiva.

\section{ET-MAP}

Unava ria ción delaan terioraplicación es elEt-Map, aplicaciónbasa da tambiénen latécnicademapa au to-organizativoo SOM.Esteproto tipoes básicamenteunsistemadecatego rización de sitiosweb. Elsistemain tentabrindar, deformaau to mática, una aproxima ción te mática ala ca te go riza cióny bús que da de in for ma ción desitios web (Chen \&. al. 1998), ${ }^{4}$ aun que también se ha im ple menta dopara repre sen targráficamentelain for ma ción que circula en los sis te mas de reunioneselec trónicas(EMS) (Orwing, Chen y Nunamaker 1997). ${ }^{5}$ El mapa en sí, es un mapa 2D y de categoría multi-capa,cuyoobjetivo finalpersigueofrecerunnavegadordeinformaciónvisual

4 H. Chen, A. L. Hous ton, R. R. Sewell, and B. R. Schatz. "In ter net browsing and sear ching: User evalua tions of ca te gory map and con cept spa ce te chniques", en Jarnal oftheAmericanSodieyforIn formationSaience, v. 49, n. 7, 1998, pp. 582-603

5 R. Or wing, H. Chen and J. A. Nu na ma ker. "Gra phi cal, Sel f- Or ga nizing Approach to clas sifying elec tro nic mee ting output”, enJaurnaldtheAmericanSodieyoflnformationSaiencer. 48, n. 2, 1997, pp. 157-170. 


\section{Métodos y Aplicaciones para el Mapeo de Intemet 95}

\section{Figura 1.}

Metáfora Visual Generada por Vi sual Site Map

Fuente:http://www.cybergeography.org/atlas/web_sites.html

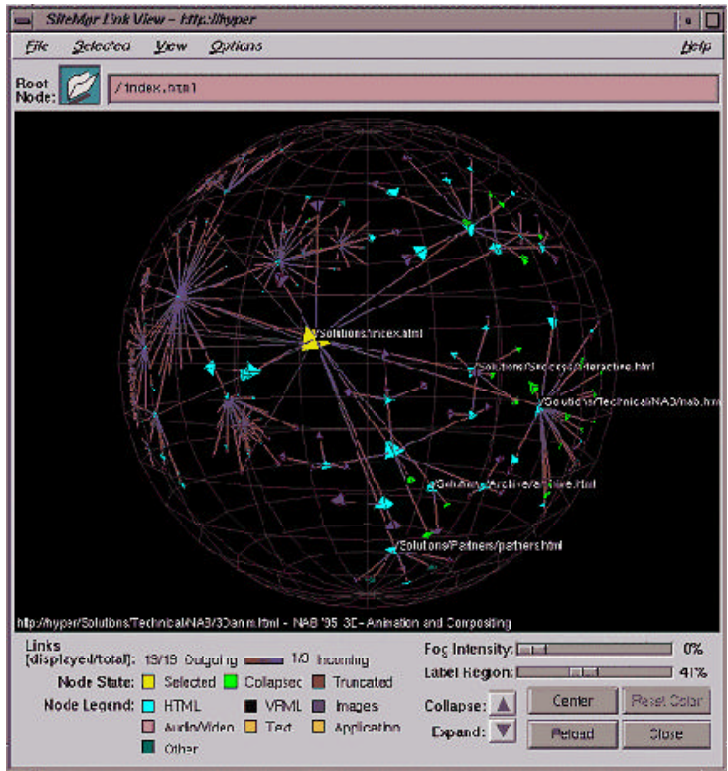

Figura 2.

Metáfora VisualGenerada por ET-Map

Fuente:http://mappa.mundi.net/maps/maps_009/

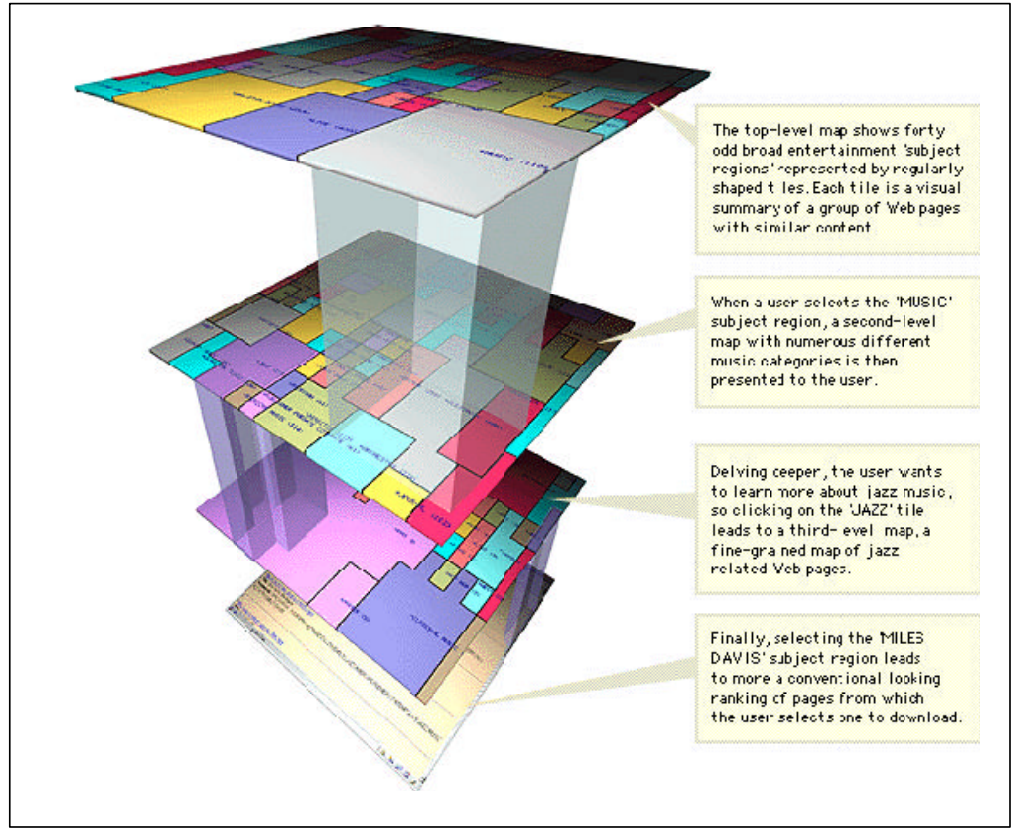




\section{InvestigacoónBiblictecoógia v. 17 N o. 35 julio/ diciembre de 2003}

intuitivo. ET-Mappuedesernavega dodeformain teractivasimplementeutilizando elratónyseleccionandolossitios deinterés.

En ET-Map,cada pa nel es un su ma riovisual de ungru po depá ginas web con con tenidosingular.Estospaneles, sesubdividenen un númerodeceldasquepresentandiferentes colores degradados, acompañados de nombres que identifican la materia de cada una de ellas. De for ma adi cional apa re ce un nú me ro en pa rén te sis que nos ofrece ráelnúmerodeenlacesdepáginaswebindividualesquerepresentacadacategoría.

Estemodeloutilizadosimportantesconceptosespaciales ensuorga nizaciónyrepresenta ción delaweb. Elprimerconcep to harárefe ren cia al ta maño delas regio nes temáticas, queestádi rectamen te relaciona doconelnúmerodepáginas webexis tentes en cada ca te go ría. Y en re la ción con el se gun do con cep to es pa cial, éste hará mención a la pro xi mi dad y se aplica rá asía re gio nes de ma te ria re la cio na das de for ma estrechaconlostérminosdecontenido.

ET-Map, como antes se señaló, es también un mapa multi-capa, con submapas que mues tran una ma yor re so lu ción de in for ma ción a tra vés de un ade cua do gra do de catego rización. Así, de esta forma, a cualquierregión de mate ria que con tenga más de200páginas web, selegene raráun mapa de segundonivelcon más ca te go rías de ta lladas. Estasubdivisión deles paciode in formaciónesrepeti da deformajerárquica y descendente(delomásgeneralalomásdetallado),siemprequeseanecesario.

Elprincipalobjetivodeesta aplica ción esgeneraruna me táfo ravisualquele facilite al usua rio una me jor com pren sión de la in for ma ción. Para ello se em plea una representaciónjerárquicadeca tego rías, divididas y frag mentadasporafinidad temática, en formadepaneles mul ticapascompuestos pordiferentesceldillas, tan tascomo temáticashaya.

De esta forma se convierte en una aplicación que le permite al usuario adoptar nuevasperspectivas de asi mila ción y co ne xión dela in for ma ción, me dian te una na vegacióninteractivaeintuitivaatravésdelasdistintascategoríasjerárquicas.

\section{ST ARLIGH T}

StarLightrepresentael primerinten to de unirunavariedaddediferentestiposconven cionales devisualiza ción de in forma ción en unúnicoeintegra do sistema dein formaciónquetienelacapacidaddeofrecerunaampliagamade funcionesanalíticas.

Lo más des ta ca do de esta aplica ción es su ca pa ci dad parain te grar una am plia varie dad de tiposes pa ciales yno es pa ciales; porunlado, tex to y da tos nu méricos, y, p or otro, coordenadasgeoespacialescomolosdatos $\mathrm{DAO}$, parade esta formanalizarlos deformaconjunta.Deestamanerala metáfo radevisualización generadamediante esta aplicación permite trabajar con extensas colecciones de información multiforma to des de una única pers pec tiva de análisis. Esto le per mi te alusua rio po dercambiar en tre una va rie dad de mo dos de análi sis (por ejem plo, el análi sis de tex to, la mineríadedatos, elanálisisgeoespacial,etcétera). 
Deestama ne ra se po sibili ta unanáli sis visual a partir de una am plia va rie dad de tiposdeinformación, de formasimultánea. Elresul tadoesunsistemacapazderealizar análisisdegrandesycomplejascolecciones deinformación.

Como se puede apreciar en la metáfora de visualización de StarLight de una comunidad web, los diver sos no dos re presen tan dis tin tas páginas web ylaslíneas que interconectanunos aotros son enlaceshipertex tuales.Loscolores delosnodosestán en rela ción conla to po lo gía de en la ce; asílas pá gi nas de origenapa re cen colo rea das de ver de, mien tras que los en la ces in ternos se re pre sen tan de azul y los ex ternos de coloramarillo.

Así, este software, mediante los mapas que genera,permite descubrir importantes relacionesamenu dosutiles enun primeranálisisy conmúltiples face tas en tre elcontenido de grandes y complejas colecciones de información. Representación visual que resultasumamenteinteractivayles permitealosusuariosmanipulargrá ficamente la informaciónen es tudio, y rápidamentepersonalizarlas demostracionesdeinformación según susnecesidades, me diantediferentes dinámicas, para queés tas puedan seranalizadasdeformaconjunta.

\section{ANÉ MONA}

Métododerepresentación desarrolladoporBenFry, investigadordelMITMedia Lab. Enestaaplica ciónlas pá ginas web in dividuales semues tran comonodosramificados en for ma de ten tá culos blan cos, de esta for ma la me tá fo ra vi sual adop ta eldibujodeunaanémona.

Este método analiza los ficheros de transacciones de los sitios web (websiteusage log y representa la actividadregistrada de forma secuencial mediante una visualización en 2D. En el mo men to que una páginaes visitada,ésta es representa da de forma au to mática en formade unnodoramifica do. Es tos nodos son co nectados conotros medianteconexionesquemues tranelenlaceindicado. Delaes tructurade enlaces, se deducela ma neraen quelos da tos sonusa dos, y de esta forma sein cre men tanlos nodosala mis mavelo cidad que las pá ginas son visita das. Deesta mane ra se pro porcionaunaherramientaquepermitelarepresenta ción en tiem porealdelusodelared.

Un dato importante a tener en cuenta es que la anémona mostrará solamente aquellas par tes del si tio que re ci ban vi si tas, de tal forma que cuan do una pá gi na deje derecibirvisitas susnodosirán dis minuyendode formagra dual hasta desapa recerv isualmente. Deesta formala anémo na podrá facilitaruna visión de talla da ymuycomple ta de lo que ocu rre en un sitio con cre to, y vi sua lizar pa tro nes y re la cio nes a través deltiempoydelasestructurasdelas distintaspáginasweb.

En esta aplicación la interacción del usua rio con la pro pia me tá fo ra de vi sua li zación co bra un pa pel cru cial. El usua rio pue de se lec cio nar un nodo para visua li zarla página web en la que se en cuen tra, pero tiene, además, la ca pa cidad de na ve gar por losnodoscircundantesalseleccionado. 
98 InvestigacoónBiblictedoógia v. 17 N o. 35 julio/ diciembre de 2003

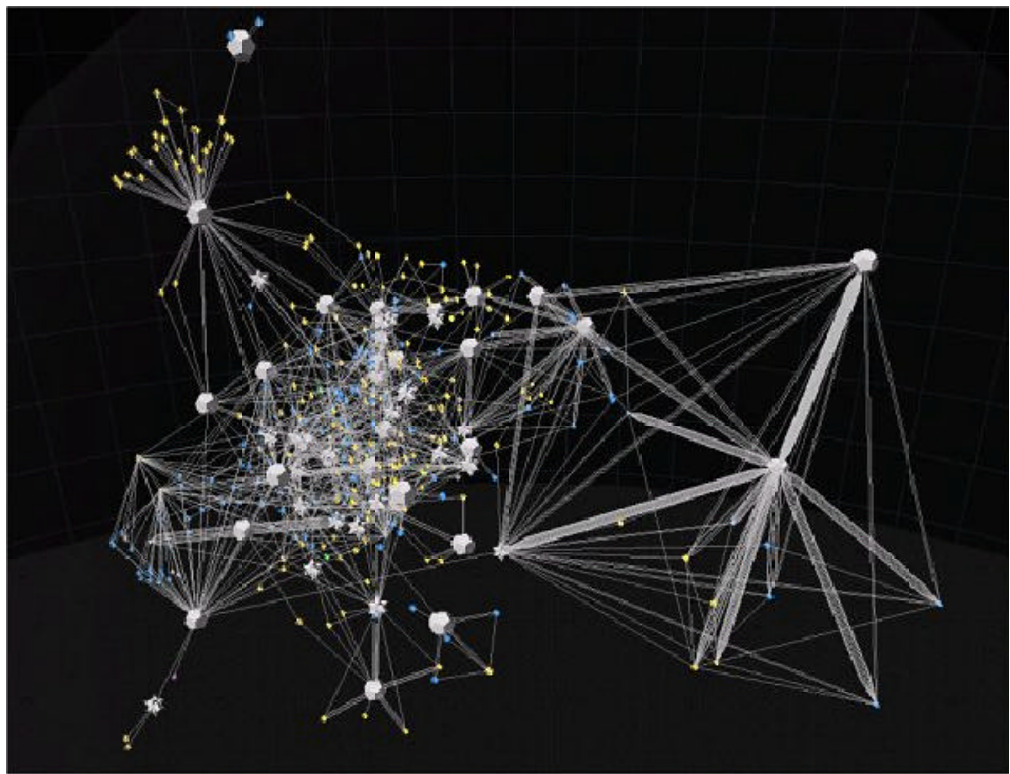

Figura 3

Metáfora Visual Generada por StarLight

Fuente:http://starlight.pnl.gov/

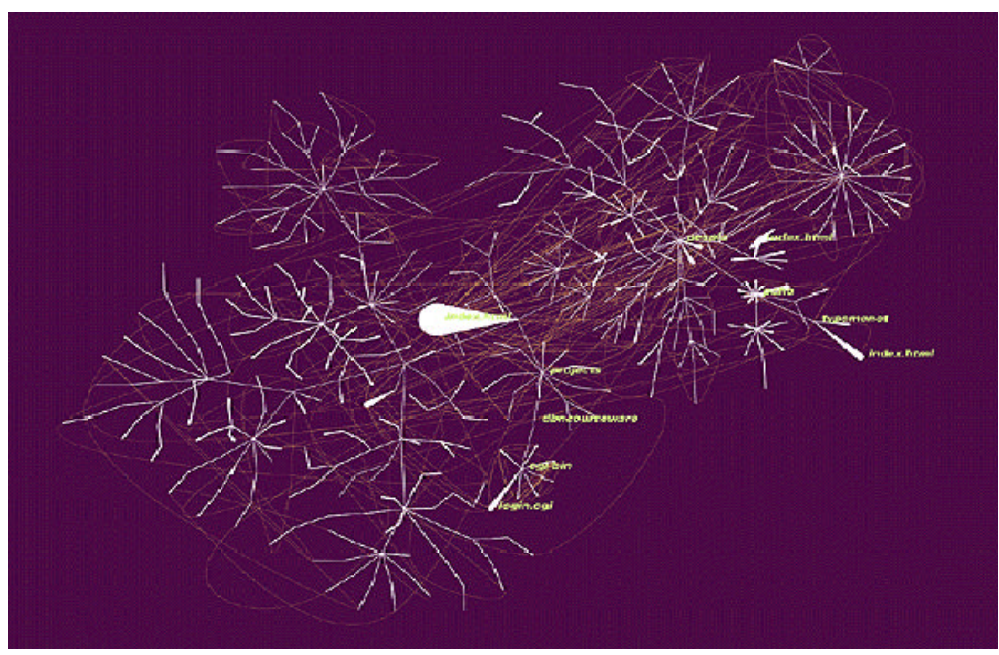

Figura 4

Metáfora VisualGeneradaporAnémona

Fuente:http://mappa.mundi.net/maps/maps_022/index.html 
Así, esta metáforaderamificacionesfacilita una navegación in tui tivaa lavezque descriptiva por la capacidad que le brin da al usua rio de po dervi sualizarunaima gen delas dis tintas rela ciones te máticas exis ten tes en el conjuntodela es truc tu rageneral de la web y le proporciona al mismo tiempo un punto referencial en la navegación dentrodelconjuntodelared.

\section{VISVIP}

VisVip es parte del paquete de herramientas de WebMetrics; software diseñado para asis tiralos expertos enusa bilidad enla labor de evaluaraplicacio nesweb. Elobje tivoprincipalde Vis Vipes visualizarda tos generadosporla he rramienta Web Vip, herramien ta que le permite a los ex per tos ge ne rarun re gis tro con forma do esen cial mente por la secuencia de direc ciones web o URLSde la ruta seguida por el usuario. De este modo re fleja la na ve ga ción efec tua da por el usua rio en el trans cur so de su vi sitaalsitioweb.

A raízdela crea ción de este re gis tro, Vis Vippermitegenerarde forma au to mática una me tá fora en $2 \mathrm{D}$ del si tio web, don decada nodo del grá fico está simboliza do por diferentes códigos de colores; el azul para HTML, el púr pu ra paralos direc to rios, el amarillo para las imágenes y así de forma consecutiva, re presen ta una pá gina web, y laslíneas representanlos linksentrelas distintaspáginas.

Ademásdelametáforagenerada, la aplicaciónpermiteunseguimien to exhaus tivo delos dis tin tos usuarioseidentifica, también, me dian te una co di ficación de colo res, las dis tin tas rutas denavega ción, yes pe cifica elco mienzoy finaldeéstas.Jun to a ello, el soft ware mos tra rá el tiem po em plea do por cada usua rio en la na ve ga ción de cada nodo en particular, con lo que pue demos trar, de forma di ná mica y si mul tánea cual quierrutadenavegación decualquierusuario enuntiempodeterminado.

De esta forma, Vis Vip se con templa comouna al ter na tiva via ble para visualizarla ac tividad delusua rioenla red y se con vierte, en una poten te herra mien tapara realizar estudios de usabilidad, así como de evaluación de semejanzasy diferencias entrelos es tilos dela nave ga ción delos suje tos. Con tribuyeasíagene rarimágenes comprensi bles de cual quier sitio web de for ma muy sim pli fica da, me dian te la po sibilidadde suprimir to doslos no dos de un tipoes pe cifica do, onodosin dividuales. Deesta forma elimina el rui do exis ten te en los di feren tes si tios web en forma deimáge nes o emails quedificultanunavisualizaciónlimpia.

\section{THEBRAIN}

Esta aplicación, considerada como un sistema asociativo de organización de información, proporciona una metáfora de vi sua liza ción que va más allá que la típica es truc tu ra de árbol, al mos trarlas co nexio nes más com ple jas delas web. Fa cili ta así 


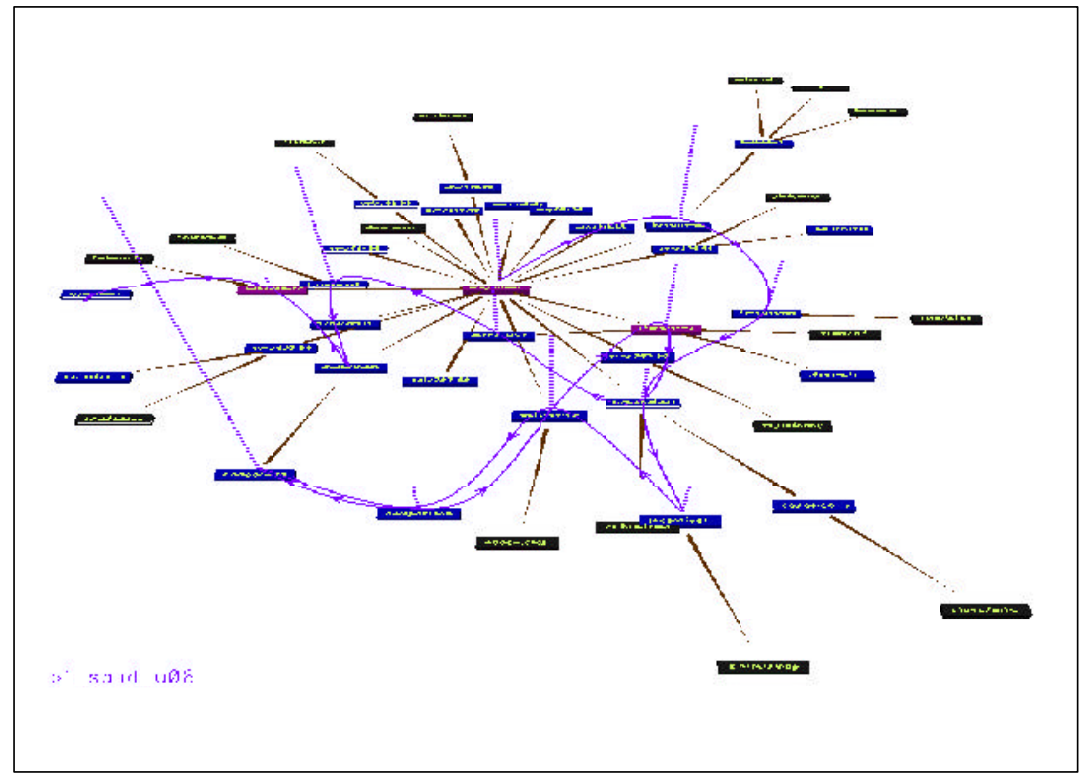

\section{Figura 5}

Metáfora Visual Generada por Vis Vip

Fuente:http://www.nist.gov/itl/div894/vvrg/cugini/webmet/visvip/vv-home.html

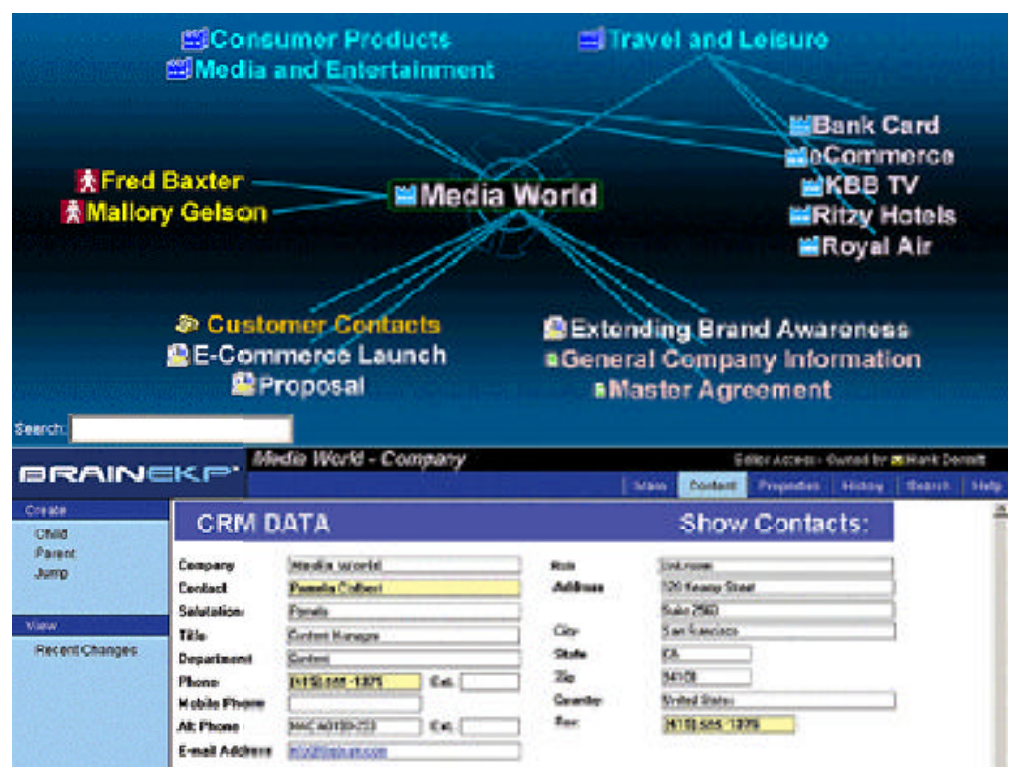

Figura 6

Metáfora VisualGeneradaporThe Brain

Fuente:http://www.thebrain.com/ 
uncon tex to visual cen tra do enlas redes de aso ciaciones lógicas existentesa travésde losenlacesdelas diferentesestructuras deinformaciónquehayencada sitioweb.

En TheBrain los ítems son denominados "ideas", las cuales pueden representar ficheros, páginasweboregistros deba sesdedatos.Lapre senta cióndeTheBrain está orga niza da en torno a una "idea" que es ta rá a su vez ro dea da por to das sus "ideas" relacionadas. Al elegir cualquiera de estas "ideas", el ítem seleccionado pa sa rá a ser el centro de la presentación y la intefaz se reconfigurará de forma automática en relación con el nuevoítem selec cio na do. Almis mo tiem po que na vega mos a través delos datos, la información mostrada en la pantalla hará siempre referencia al dato seleccionado en cada momento, lo que además nos permitirá, seguir una ruta de una "idea",eirnavegandoentreellas dentrodegrandescontextosinformativos.

Estemodelode co nocimien toproporcionauna visualiza cióndees tas relaciones tal y como és tas exis ten en el mun do real,y permi te una com pren sión total delain formaciónmediantelacreacióndediferentes formas deaccesoalainformaciónclave.

Se tra ta porlo tan to de una so lu ción ba sa da ex clu si va men te en la web, conlaidea de compartir y colaborar en la gestión y análisis de grandes cantidades de informaciónypermitirlealusuarionavegarporunespacio que muestraenlaces yafinida des a travésdelposicionamientorelativodelosdiferentesítems.

Paraellogeneraun mapa de conocimien to visual formado por una in ter faz visual de navegación in tui tiva. Estolepermiteal usua rio fi nal ela bo rar me tá fo ras visuales personalizadasy acceder a datos universales, además de realizarbúsquedas contextuales.Estainterfaz, me dianteelproce sodevisualizacióny con tex tualización faculta alusua rio paraverto das las contin gen cias relevantes, nece sa rias parala toma de de cisiones. Para ello hace va ler la re pre sen ta ción de la in forma ción den tro de su con texto, conlo quele propor cio na al usua rio un cua dro visual revela dor deloexis ten temás allá delain for ma ción que se convierte en el hilo con duc torquelepermi ti rá al usua rio en ten derlaim portan cia delain for ma ción analiza da. Estole pro porcio na al usua rio un am bien tein te gra do para crear in forma ción. ypara acce deryvalorarésta dela mis maformasencilla.

\section{T OUCH GRAPH}

Tou chGraph es unaherramien ta aplica da ala nave ga ción visual delas bases de da tos de los mo to res de bús que da, como Goo gle, Ama zon... con el fin de ex plo rar los enlaces existentes entre los distintos sitios web relacionados. El resultado de esta aplicación esla ob ten ción de una me tá fora vi sual de una mapa di ná mi co y nave gable de nodos y links Donde cada nodo está claramente identificado y aporta informacióndetalladadecadasitioweb.

Tou ch Graph proporcio na una granca pa cidad de ma nio bra. Las re des son da das como gráficos interactivos que se prestan a una gran variedaddetrans formaciones. Ajustandolaimagen seleccionada, elusuario puedenavegara través de gran desredes 


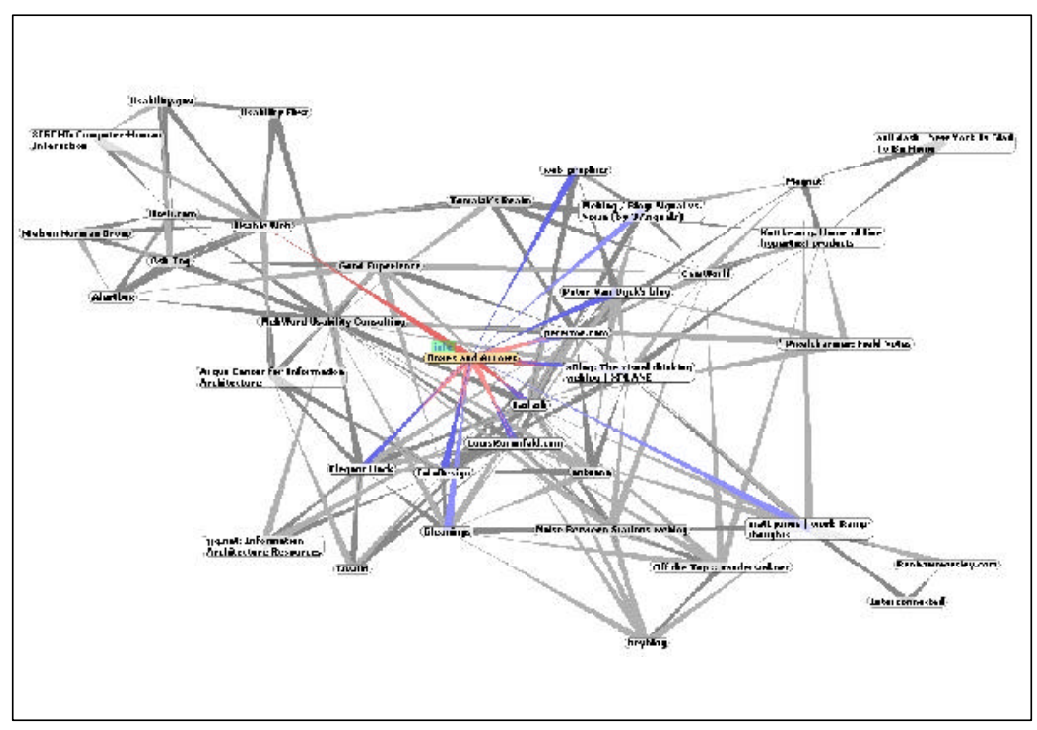

Figura 7

MetáforaVisual Generada por TouchGraph

Fuente:http://www.touchgraph.com

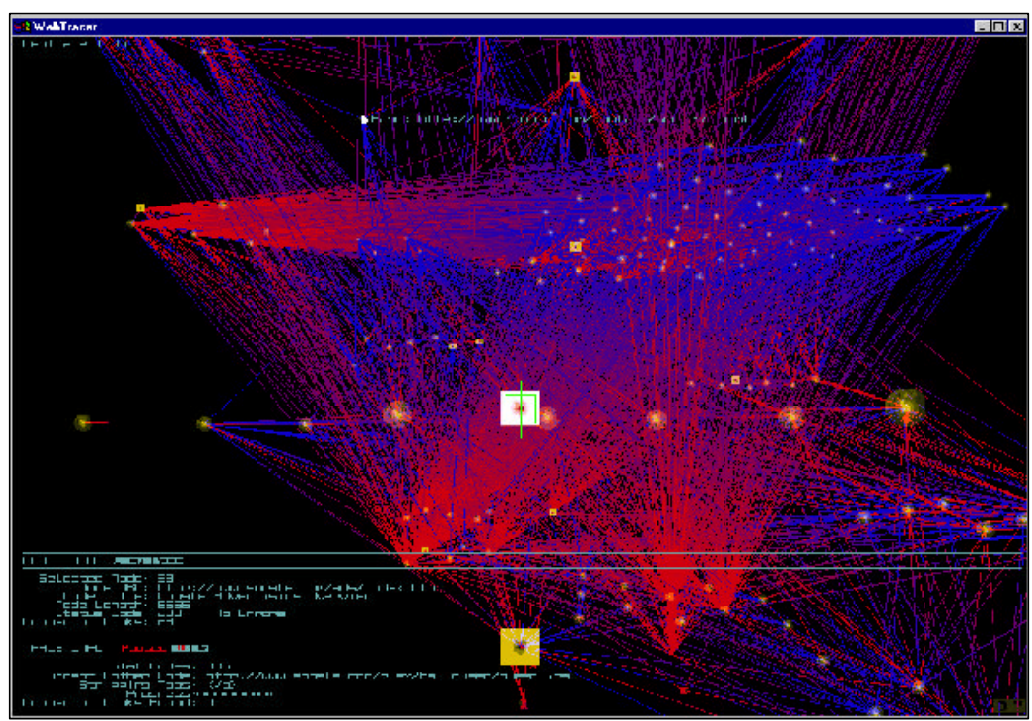

Figura 8

Metáfora Visual Generada por WebTracer

http://www.nullpointer.co.uk/-/webtracer.htm

http://www.nullpointer.co.uk/-/webtracer2.htm 
y explorar diferentes formas de visualizar los componentes de la red en la metáfora proporcio na da por el soft ware. De esta for ma el usua rio ob tie ne una na ve ga ción dinámicaeintuitiva.

Así, TouchGraph se constituye en una herramienta ideal no sólo para descubrir los enlaces relacionados con el sitio web seleccionado, sino tam bién para descubrir enlaces con otros sitios webquea prime ravista no pre sentaban semejanzaorelación algunaconlawebseleccionada.

\section{W ebT racer}

Web Traceres un pro yec to ba sa do en lain ten ción de visualizarla es truc tu ra dela web. Esta aplica ción mues tra, me dianteuna me tá foravisual,la es truc turagene rada porlosenla cesweby la representa como un dia gra ma molecularen3D, don delas pá ginas web son re pre sen ta das como no dos (áto mos) y los dis tin tos en la ces que in terrelacionan aunas con otrascomo ca denas (enlaces ató micos) que conec tan esos nodosen tresí.

Lasbases de WebTracerseapoyan en dos pilares fundamentales para elanálisis de es tas es truc tu ras y la pos teriorgene ra ción dela me tá fo ra visual; una arañay unvisualizador. La ara ña se apli ca para vi si tarlos di fe ren tes si tios web y para ge ne rar ma pas de archivos que contengan in for ma ción sobre sus en la cesin ternos. Mien tras que, el visualizador, permitirá representar esos mapas en $3 \mathrm{D}$, mediante una metáfora con apariencia de estructura molecular donde aparecerán tanto páginas web como sus respectivosenlaces.

Dentro del contexto de WebTracer, la estructura de los sitios permite que puedan serexamina dos des de cual quierán gu loy dis tancia, como si fue ran moléculas. Los nodos pueden ser seleccionados para obtener mayor información sobre su contenido, además de para acceder de forma directa a la página correspondiente. La estructura molecular del sitio refleja la secuencia de enlaces dentro de la página, y también la estructurain ternadelsis te ma de fiche ros delservidorweb.Deestaforma Web Tracergeneraráundiagramamolecularinteractivoqueseráúnicoparacadasitiovisitado.

Setratadeunaherramientainteractivapensadaespecialmenteparavisualizarlaweb ypermitirlesalosusuarios recopilarin formación sobrelos dis tin tos sitios web at ravés desuestructura, locualselogra analizan doprio ritariamenteeldiseñoylaconstrucción delos sitios.

\section{Z-DIAGRAM}

Z-Diagramesunainnovadorametáforavisualpararepresentarestructurasdesitios web y fue de sa rro lla do por Dyna mic Diagrams Inc. En esta aplica ción, tan to la es tructu raje rárquica como elvolumen depáginas, la pro fun didad delos enla ces einclu so el acceso correc tosontodoscla ramenterepresentadosusan douna combinacióndeuna 
distribuciónespacialjuntoconunacodifica ciónmediantecolores.Las distintaspáginas web, represen ta das en for ma de pe que ñas tarjetas, seorde nan deiz quierda de rechay mues tranla pro gre siva pro fun didad delos en la ces den tro dela pá gina deinicio. De forma simul tánea, los diferentes coloresdelascarpetasdesignandiferentes nivelesdeacceso.

En esta aplicación las tarjetas serán or de na das de tal for ma que cada capa re presen ta rá un nivel de pro fun di dad den tro del si tio web. To das las pá gi nas (hijas) se rán repre sen ta das en el mapa a una mis ma dis tan cia, con lo cual se evita cual quier tipo de tras lapamiento.Laspáginas enlaza das aotras se represen ta rán detrás deés tas en filas paralelas. El número de filas le fa cili ta ráal usua riouna visiónins tan tánea ein tuitiva delvolumendepáginasexistentesdesdesulocalización.

Los códigos de co lo res se usa rán tam bién para fa ci li tar laiden ti fi ca ción de la estructurajerárquica; de esta forma las páginas (hijas) inmediatas seránrepresentadas en color verde y las páginas (nietas) subsecuentes serán representadas en azul. Por debajo denues tratarjeta delocalización, laspáginas an tecesorasserán mostradas en colornaranjaydelinearánla rutadenavegaciónhastalapáginaprincipal.

Enesta aplica ciónelusua rio po dráin te rac tuarconelmapageneradode diferen tes formas. En primerlugar,alpasarelcursorso breuna tarje ta segene raráel realzamiento de la mis majun to con la mues tra del título de la tarje ta es pe cifica da. Aun quetambién podrán navegar dentro del propio mapa seleccionando las diferentes páginas. Para ello ten drán que ha cer clic so bre las tar je tas con una ba rra ne gra y és tas se re ordenaránellasmismasenunatransiciónlinealanimada.

Estaca rac te rís tica ha di feren ciadoal dispo sitivo comoelmásin novadory efectivovisualizadorinteractivodesitioswebdisponibleactualmente.

\section{H YPERBOLIC TREE}

Esta aplica ción es una me tá fo ra grá fi ca que per mi te la na ve ga ción y el análi sis de grandes estructurasweb je rárquicas, me diantelageneración de grá ficos dirigidosen un espacio tridimensional. Para ello emplea la técnica de visualización de "ojo de pez" con el fin de pro por cio narde forma simul tá nea tan tolos de talles lo cales como elcontex toglobaldelaestructura.

Este tipo dein ter fazhasidoaplicadoparticularmente enlavisualiza ción de sitios web.Sumodo deusoes bas tan te sen cillo ein tuitivo.Se selec ciona elnodocentraldel ár bol, el cual se pue de moverde su si tio y ori gi nar co rri mien to en la re pre sen ta ción; de esta for ma van apa re cien do nue vas partes de la es truc tu ra que no se veían, al tiempo que otras se van escondiendo. Así, pues, podemos “jalar”larepresentaciónpara que aparezcan aquellos nodos que van desapareciendo. Cuando se detecta un nodo relevante, bas ta condarleundoble clic para ac ce deral tex to completodelapáginaw eb (enla ventana contigua). Me dian te los bo to nes que se encuentran abajo a laiz quierda es posible cambiar el tamaño de las fuentes de las etiquetas, contraer-expandir 


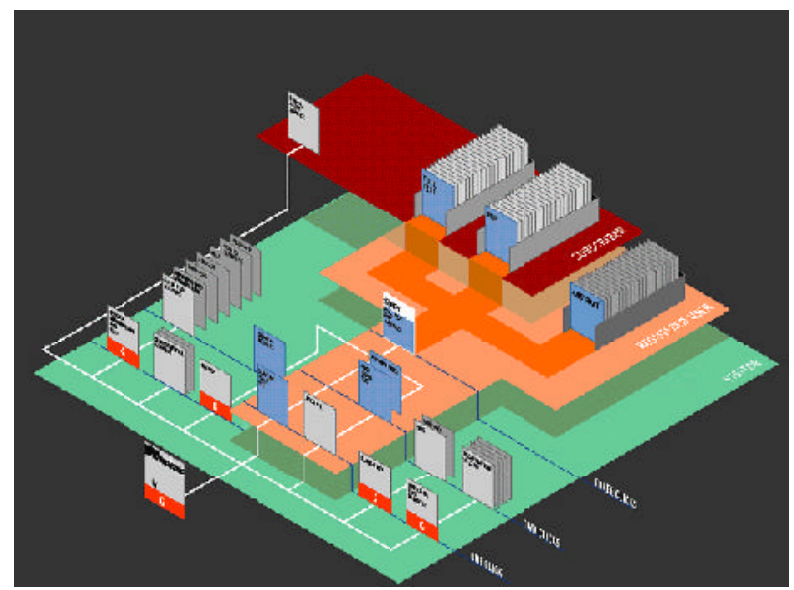

Figura9

Metáfora Visual Generada por Z-Diagram

Fuente:http://mappa.mundi.net/maps/maps_006/

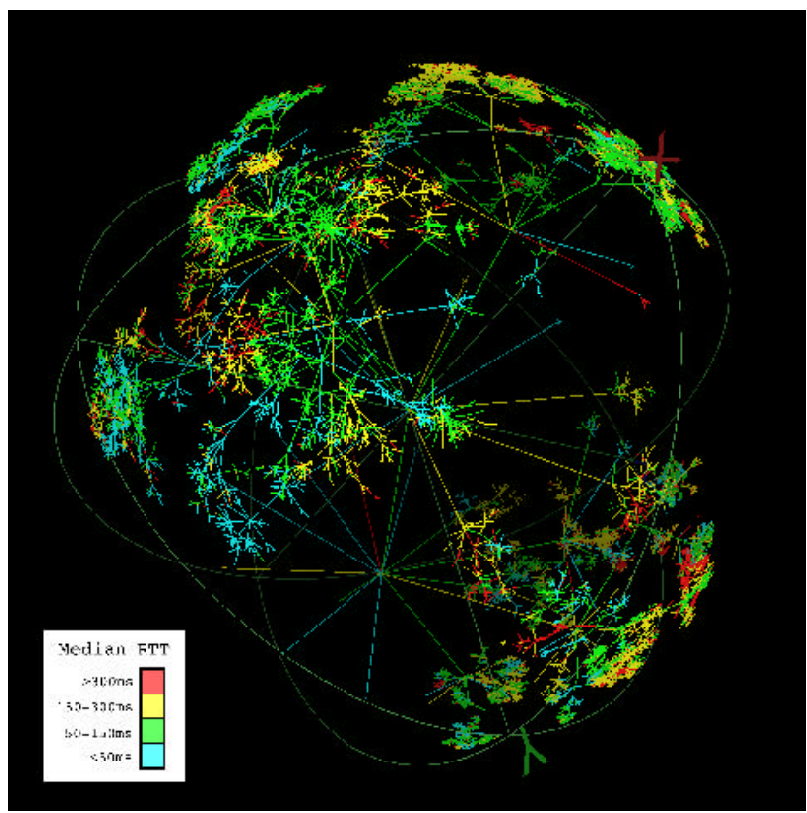

Figura 10

Metáfora VisualGenerada por Hyperbolic Tree

Fuente:http://www.caida.org/tools/visualization/walrus/ 
larepresen ta ción, volveralpun to de origen, etcétera. Deesta for ma la me tá fo rama neja bien la relaciónfoco----+contexto, mediante la cual puede entrar en detalle sin perderelcontroldelaestructura.

\section{H YPE RSPACE}

Esta aplicación de HyperSpace consiste en una interfaz desarrollada por el Advanced Interaction Group (AIE) de la Universidad de Birminghan, y su objetivo es organizartridimensionalmentelainformaciónatravés deenlacesdelas páginasweb.

Para ellorepresentalaspági nas me dian te es fe ras de diferentes tamaños ylosenla cescon sim pleslíneas. De esta ma ne ra con for ma una se rie de ga la xias y cons te la ciones de es feras de dis tin tos ta maños uni das por líneas, lo quepresen ta una me tá fo ra espacial típica. Cada esfera se com por ta como un pla ne ta: cuan do más gran de, más atracción ejercesobrelasesferasmáspequeñas.

La me tá fora deplanetas se pue de ob servaren una pequeña ven tana mien tras que enotrase tiene el navega dorweb funcionando. Cuandounaes feraes seleccionada, la páginawebcorrespondienteapareceenlaventanadelnavegador.

\section{SURF 3D}

Surf3D es una po ten te aplicación deúl ti magene ra ción para la bús que day visualizaciónde da tos. La representacióngráficagenerada porestesoftwarepo sibilita optar en tre ochometáforasgrá ficas, ymues tracómolaweblocalizada es representada medianteelusodecolores, tamañosyvaloresnuméricospararepresentarsurelevancia.

Uno de los principalesbaluartes de Surf3D es su funcionalidadparatras ladarlos procesos de búsqueday sus re sul tados a me táforas visuales, loquelograme diantela utilización y aplicación de gráficos que automatizan los procesos de investigación web y establecen controles de alerta convencionales a la vez que ofrece informes HTML y combina varios elementos, tales como agentes, buscadores y tecnología software.

Jun toalametá foravisual generada porla aplica ción apa receránuna se rie dein formes rela cio na dos con ella mis ma que se mos tra rán en la par te in fe rior dela pan tallay queharánreferenciaaladefinición dela webdondeapa recerálainformación relacionada en distintos directorios; también incluye y excluye condiciones y busca términosin tro du ci dos en los bo to nes debúsque da que se en cuen tran tam bién en la parte inferiordelapantalla. 


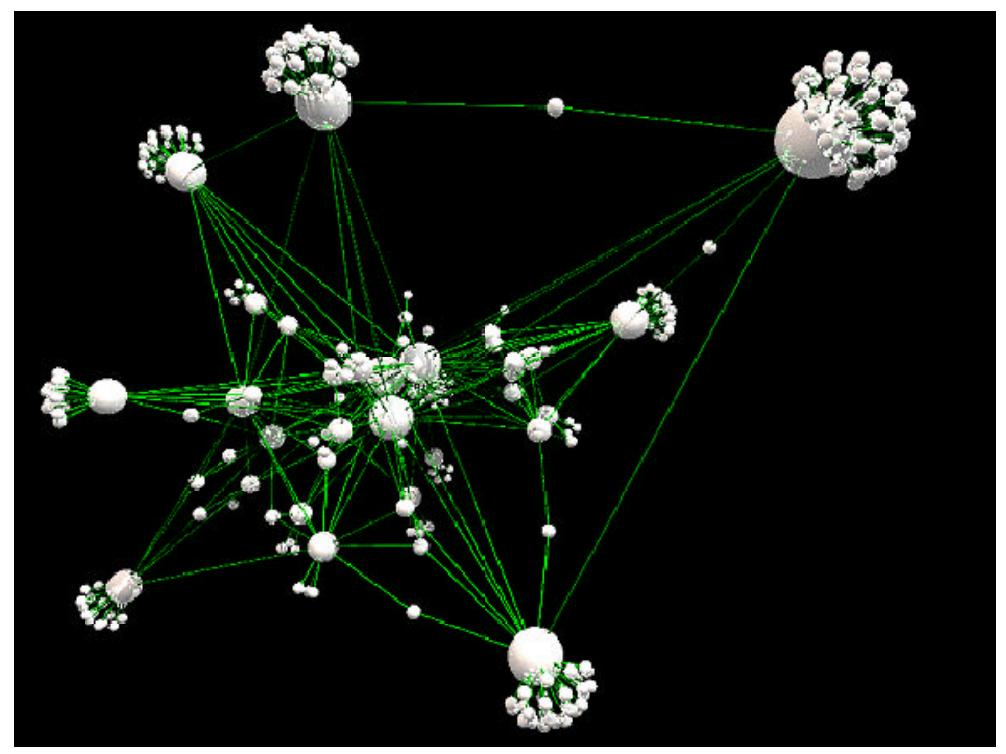

Figura 11

Metáfora Visual Generada por HyperSpace

Fuente:http//www.cs.bham.ac.uk./ amw/hyperspace/

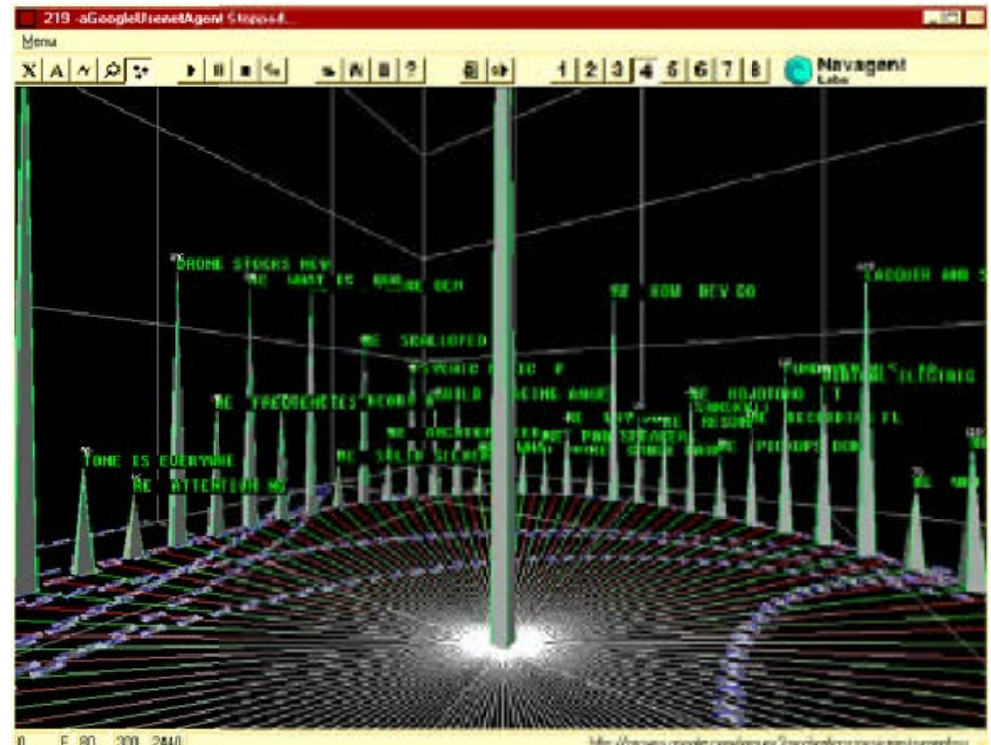

Figura 12

Metáfora VisualGenerada por Surf3D

Fuente:http//www.navegent.com 


\section{INTERNET CART OGRAPHER}

Setratadeunsistemaque observa au to mática men telasnavega cioneswebque es tán ha cien do uso de In ternetExplorero Nets ca pe Naviga tor. A raíz de esta ob serva ción, el software ge ne rauna aplica ción don delos sitios web son cla si fica dos den tro deunaje rarquía de 500 ca te go rías dis tin tas ba sa das enlos términos dis tribuidosdentro de los documentos y que son simultáneamentemapeados dentro de un espacio semánticobasadoenenlaces.

En Internet Cartographer, la representaciónvisual estarácompletamentebajoel con trol del usua rio, pues to que esta apli ca ción le per mi te man teneruna vi sión comple ta de su his to rial de na ve ga ción enla red. Ade más capa ci ta al usua rio para rea lizar bús quedas en su his to rial de na ve ga ción, porel título delas pá ginas o porlas palabras clave delos contenidos deés tas. De esta formavisua liza sitios relaciona dos porenlaces (físicos), contenidos y fe chas. También permite fil trary eli minaren la ces, de formadirectamedianteelusodepalabrasclave.

Pormedio delasopciones de en fo que y fil tra do, el mapa es ma ni pu la ble usan do una única visión macroscópica que muestra simultáneamente una visión global de to dos los si tios, en la ces y tér mi nos, mien tras que rea jus ta la pers pec tiva delusua rio en la visión prin cipal.Los usua rios, a su vez, pue den de fi nir fil tros que pre ven gan acciones so bredis tin tos sitios basa dos en pa trones URLs, o ba sa dos en tér mi nos dentrodelosdocumentos.

Esta aplica ción les fa cili ta ráa los usua rios a ges tio nar el gran volu men dein for ma ción que visualizan enlaweb. Para ello mues tran los si tios web vi si ta dos y la asigna ción de categorías para estos sitios a través de diferentes tipos de visualización, concretamente, median te tres ven tanas de visualiza ción: el mapade visualización, el cua drode navega ción de la propia visualiza ción y elárbol de visualiza ción. El mapa de vi sualización muestra los sitios visitados y opcionalmente los enlaces y las palabras asociadas con cada uno de ellos. El cua dro de na ve ga ción mues tra el mapa com ple to, y pro porciona asíla po sibili dad de seleccio nar las di fe ren tes partes de éste para aplicaruna amplia ción de ellas y permi tiren traren más de talle. Y elárbolde visualiza ción muestra un árboldeca tegoríasysitiosweb.

De esta for ma sele fa cilita al usua rio la visua liza ción de gran des y com ple jos conjun tos web, a la vez que sele fa cili ta la presen ta ción y com pren sión delas dis tin tas relacionesexistentesentrelosdiferentessitiosweb

\section{Internet Mapping Project}

Se tra ta de otras de las líneas de tra ba jo im pli ca das en el es tu dio y de sa rro llo de mapas relacionados con los flujos de información que se producen en la red en tiemporeal; concreta men te del análi sis delcre cimiento, dela cober tu ra y del tamañodeInternet. 


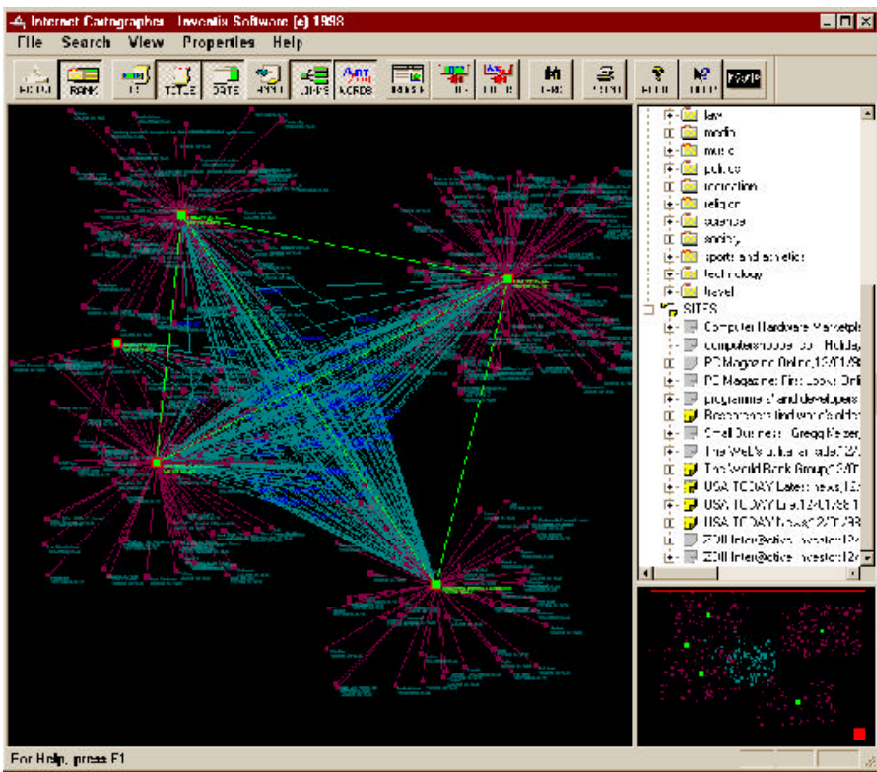

Figura 13

Metáfora Visual Generada por InternetCartographer Fuente:http://www.inventix.com/

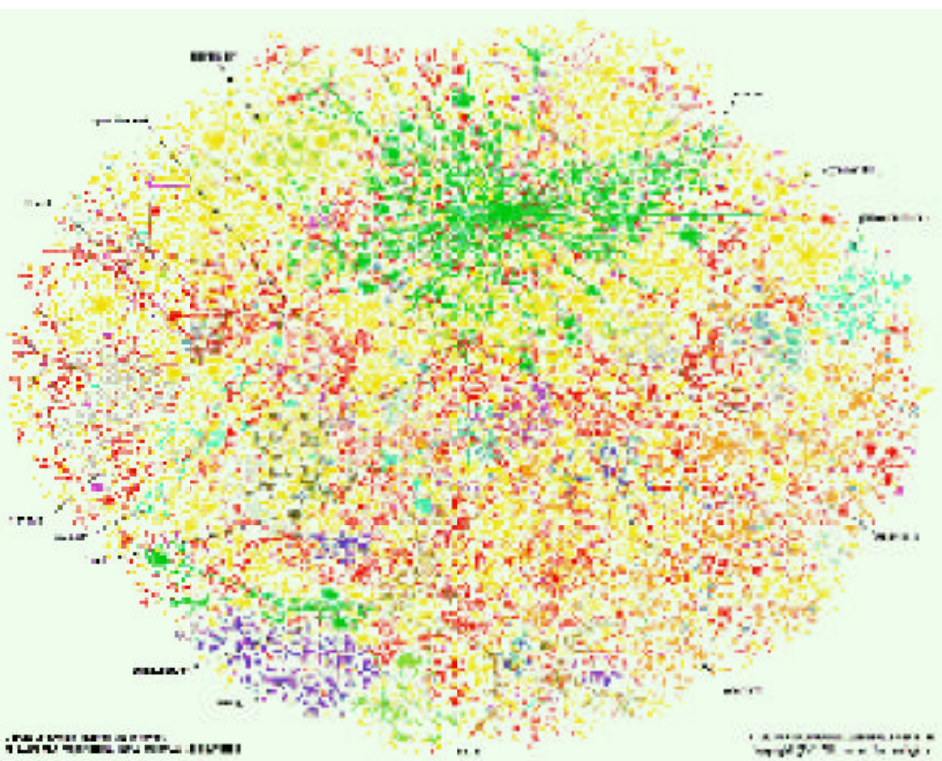

Figura 14

Metáfora Visual Generada por Internet Mapping Proyect

Fuente:http://research.lumeta.com/ches/map/gallery/index.html 
El mapa generado por esta aplicación se basa concretamente en sondas de rutas frecuentes enredesIP, cadaunadelascuales regis traentidades Internet.

Con es tos da tos se cons tru yenár bo les que mos tra rán los sen de ros de la ma yo ría delasredesdeInternet.

Son es truc tu ras que, jun to con los ma pas gene ra dos, ayu da rán al usua rio a vi sualizar elementos, a focalizarpuntos dein te rés, y a lo calizar even tos que jus tifi quenun análisis más detenido. Una vez que la presentación es calculada, el mapa puede ser elaborado con el fin de mostrar un número de elementos; direcciones IP, regiones geográficas, etcétera.

Elobje tivo finalde toda esta ac tividad es crearun atlas deInternet. Sein tenta facilitarle al usuario final la visualización de la red, con la intención de hacer más compren sible el di se ño or gá ni co dela in for ma ción dela web y de re su mir de for ma di ná mica y accesible la compleja actividad de las interacciones en línea. Para ello, proporcionaunesquemadelatotalidaddeInternet.

\section{CONCLUSIONES}

Actual mentelas diferentes aplica ciones para elma peo deInternetseen focancasi de ma ne ra ex clu si va a des cu brir la to po lo gía dela red, y de for ma par ticu lar el crecimien to, la coberturay el ta mañoque hapropiciado su trans formación enla ac tual red de redes. La principal finalidad es ayudar a comprender las implicaciones sociales, políticasyeconómicas delciberespacio,y revelarlasinterrelaciones exis ten tes en tre lasdistintassubredes.

Aun que hoy por hoyes tas he rra mien tas de aná li sis y sus aplica cio nes son sim ples desarrollos experimentales y prototípicos que aún no han cristalizado en aplicaciones comerciales, im plicanunamplioabanico de posibilida des en eles tudio delasorganizacionesyen particularenelcampodelas relacionesinter-organizativas. Constituyen también una herramienta de poderosa utilidad para la operación de datos rela cionales, la compren sión delospa tro nes es truc turales delas rela ciones en treinstitucionesyelanálisisderedes sociales.

Estas herramientas tendrán una doble finalidad; ser instrumentos de análisis y también interfaces dinámicas e intuitivas que facilitarán la navegación del usuario. De estemodolas dis tintas me tá foras genera das pores tas aplica ciones se ránalgo más que sim ples ele men tos vi sual-in forma tivos. Con ellas el usua rio ten drála ca pa ci dad de interactuar mediante distintas opciones con el gráfico y obtener la información necesaria para mejor com prender los diver sos es pa cios dein te rac ción exis ten tes en laredynavegarconmás facilidad.

La mayoría de estas aplicaciones muestran todavía representacionesde espacios elec trónicosabs trac tos si mila res a un dia gra ma de flujos, cu yos es fuerzos se cen tran principalmente en incursiones, muy tímidas aún, en la construcción de una visión global del tráfico de la red. Sin embargo, el simple hecho de su materialización y la 
con secu ción de los prime ros re sul ta dos en forma de ma peos Web presen tan una al terna tiva de fu turo fun da men ta da en el desa rrollo de unapoten te aplicación paravisualizarInternet, que ten drála ca pa cidaddepro porcionarin forma ción muyvalio sa que podráaplicarsedes de eldiseño dein fraes tructu rasenlared, pa sandoporelanálisisdeflujos dein formaciónentreins titu ciones, personas, países, etcétera, has ta el estu dio de usa bilidad delared me dian te el análi sis dela forma de na ve ga ción delos distintosusuarios.

\section{BIBLIOGRAFÍA}

CARD, S. K., MACKINLAY, J. D. and SHNEIDERMAN, B. RegdingsinIn formationV isualization:UsingV isionToThink. Morgan Kaufmann, 1999.

CHEN, H., HOUSTON, A.L., SEWELL, R. R., and SCHATZ, B. R. “In ternet browsing and sear ching: User eva lua tions of ca te gory map and concept space techniques", en Jarmal of theAmeican Soidy for Infomation Siene, v. 49, n. 7, 1998, pp. 582-603

DODGE, M., KITCHIN, R.. Atlas of Cybaspace Addison Wesley, 2001

DÜRSTELER, Juan Car los.Visualizacoóndelnformacón Ediciones Ges tión 2000.com, 2002

HERRERO SOLANA, Víc tor. Moddosderepresentacoónvisualddainformacoón bibliogáfica: aproximaaiones miltivaniantes y conexionistas Granada. 2000. (Tesis Doctoral)

KOHONEN, Teuvo. Seff-aganizingmaps Berlin: Springer, 1997

LIN, Xia. "Searching and browsing on map displays". Procedings of ASIS95. Chicago, October 1995. http://www.uky.edu/ xlin/asis95.htm

--. "Graphical table of contents". Digital Library'96. http://www.uky.edu/ xlin/DL96/DL96.htm

LIN, Xia. "Map dis plays for in for ma tion re trie val", enJarmal fftheAmeicanSociey for Infomation Säence(JASIS). v. 48, n. 1, 1997, pp. 40-54

MOYA ANEGÓN, Fé lix de, HERREROSOLANA, Víc tor y GUERRERO BOTE, V. "La aplicación de Redes Neuronales Artificiales (RNA): a la re cu pe ra ción delain for ma ción", enAnuanioSOCADIdeDoamentacióne Informaión Barcelona: Sociedad Catalana de Documentación e Información, v. 42, n. 2, 1998. 
MOYA AnEgón, Félix de, Herrero SolanA, V., GuErrero BOTE, V. "Virtual Reality interface for accessing electronic information", en LibraryandInfomationRescenchNenss v. 22, n. 71, July, 1998, pp. 33-39

--, y HERRERO SOLANA,V. 'Inves tigaciones encurso sobrein terfaces grá fi cos en dos y tres di men sio nes para el ac ce so a la in for ma ción electrónica”, en Cuademos deDoumentacónMultimedianº 8, 1999

ORWING, R., CHEN, H. and N UNAMAKER,J. “A Graphical,Self-Organizing Approach to clas sifying elec tro nic mee ting output", enJarmal of theA maican Society of Information Saiencev. 48, n. 2, 1997, pp. 157-170.

TUFTE, Edward. EnvisioningInfomation Graphics Press, Cheshire Connecticut, 1990.

WHITE, H. D., MCCAIN, K. W. "Visualization of literatures", en M. E. Wi lliams (Ed.),AnnualRevienofInformationScienceandTednology. Vol. 32, pp. 99-168, 1997. Medford, N J.

WILLIAMS J. G., SOCHATS K. M., and MORSE, E. Visualization volume 30, pages 161-207. Information Today, Inc., Medford, NJ, 1995. 